\title{
Feasibility of evaluating effects of muscle fatigue on postural stability and muscular activation of the supporting leg in soccer power kicking
}

\author{
JÚLIA A. OLIVEIRA ${ }^{1}$ | CAROLINE R. DE SOUZA¹ | CARLA D. P. RINALDIN² | DANIEL B. COELHO ${ }^{1,3}$ | LUIS A. TEIXEIRA ${ }^{1}$ |
}

${ }^{1}$ Human Motor Systems Laboratory, School of Physical Education and Sport, University of São Paulo, SP, Brazil.

2 Graduate Program on Health Technology, Pontifical Catholic University of Paraná, PR, Brazil.

${ }^{3}$ Biomedical Engineering, Federal University of ABC, São Bernardo do Campo, SP, Brazil.

Correspondence to: Júlia Ávila de Oliveira, Av. Prof. Mello Moraes, 65. Cidade Universitária, USP, São Paulo, SP. BRAZIL. 05508-030

email: julia.avila@usp.br

https://doi.org/10.20338/bjmb.v13i5.146

\begin{abstract}
HIGHLIGHTS
- Effect of muscular fatigue on dynamic balance and muscular activation in a kick task.

- Muscular fatigue failed to lead to functional changes in a specific soccer task.

- Neuromuscular adaptations allow for maintenance of balance stability while kicking.
\end{abstract}

\section{ABBREVIATIONS}

APAs anticipatory postural adjustments

$\mathrm{CoP} \quad$ center of pressure

EMG electromyographic

MG medial gastrocnemius

$\mathrm{PL} \quad$ peroneus longus

SD self-declared

SOL soleus

TA tibial anterior
BACKGROUND: Muscle fatigue accumulated during a soccer game can be a critical element to athletic performance.

AIM: The aim of this study was to analyze the feasibility of evaluating the effect of local fatigue on balance and muscular activation of the support leg in soccer players when performing power kicks.

METHOD: Six soccer players were evaluated in the kicking task, supported on a force platform. The ball was stabilized on a base beside the force platform by two elastic strings attached to fixed points on the floor. The variables analyzed were: CoP displacement amplitude; activation magnitude of the soleus (SOL), medial gastrocnemius (MG), tibial anterior (TA), and peroneus longus muscles (PL) for three intervals: 200 ms prior to foot-ball contact, and between 30-60 ms and 80-135 ms after foot-ball contact; co-contraction between the MGTA and PL-TA muscles; and peak velocity of the kicking leg. Muscular fatigue was induced by means of repeated oscillations of the kicking leg until exhaustion.

RESULTS: Results indicated that fatigue failed to affect either velocity of the kicking leg or postural stability while kicking. Electromyographic analysis revealed that induced fatigue decreased activation of the medial gastrocnemius muscle and increased activation of the soleus muscle following foot-ball contact. CONCLUSION: These results demonstrate the feasibility of analyzing the effect of local fatigue on dynamic balance and muscular activation in the performance of a power kick.

\section{INTRODUCTION}

Postural stability has been shown to be determinant in soccer player performance ${ }^{1}$. Postural instability is one of the intrinsic factors that may lead to increased risk of injury? Ankle sprains have been cited as one of the most common musculoskeletal injuries among athletes who rely on sudden stops and changes of direction, like in a soccer game ${ }^{3}$. More specifically, an epidemiological study on soccer injuries showed that $17 \%$ of injuries are lateral ankle sprains ${ }^{4}$. Among ankle injuries in soccer players, $48 \%$ occur at the end of each half of the match ${ }^{5}$. It has been speculated that alteration in muscle responses caused by local fatigue may explain the increase in lower limb injuries among athletes $3,6,7$. The fatigue process induces exaggerated joint stiffness and delayed automatic postural responses by reducing spinal reflexes ${ }^{8-10}$, compromising motor control performance in reactive postural responses to perturbation ${ }^{9,11-13}$. When an individual is exposed to stressful tasks, motoneurons become less sensitive to synaptic entry, degrading proprioceptive afferent feedback from the muscle spindles, and efferent information 
becomes insufficient, compromising muscle contraction latency in several tasks ${ }^{14}$. As the literature has shown that the postural control of soccer athletes is preferably regulated by proprioceptive pathways, which are impaired by fatigue, it is possible to assume that delayed and decreased postural muscle recruitment may lead to a higher incidence of ankle injury in this condition.

Some studies have evaluated whether soccer-induced fatigue alters postural control, and may be a potential cause of the high incidence of ankle injuries in players $7,15-17$. However, results are controversial. In addition to using different protocols for fatigue induction, these studies used different mechanisms to measure postural stability, with scarce concomitant assessment of balance related to muscle activation parameters and postural control strategies. The effect of fatigue on postural control is influenced by the parameters of muscle location, decreased muscle strength, exercise intensity and duration, muscle action, and number of affected muscles ${ }^{11}$. Different compensatory postural strategies are used to reduce the perturbation of postural control caused by general or local muscle fatigue ${ }^{11-13}$, but no studies were identified in the reviewed literature evaluating the influence of the activity of different ankle stabilizer muscles and compensatory strategies on postural control after fatigue induction in soccer players.

Controlled studies about the effect of fatigue on the postural control of soccer athletes are still lacking in the literature. It would be of interest to adjust a fatigue-induction protocol to resemble the demands of the game, or induce postural perturbations that alter neuromuscular control to analyze the effects. It is also necessary to choose postural tests that resemble the movements used in the sport modality. In addition, postural control should be evaluated in conjunction with muscular activation to better understand the effects of fatigue on postural control and the possible use of compensatory strategies. The aim of the present study was to analyze the feasibility of evaluating the effect of muscle fatigue of the supporting leg in soccer players on postural stability and activation of the stabilizing muscles of the ankle in the task of kicking a ball, with similar mechanical requirements as in a real game situation.

\section{METHODS}

\section{Participants}

Six male university soccer and/or futsal athletes, right-footed for kicking (selfdeclared), age range 19-27 years $(M=22.00, S D=2.68)$, participated voluntarily in this study. As inclusion criteria, participants were required to have had no ankle sprain in the supporting leg in the previous six months, have participated in regular training in the previous four years, with at least three training sessions per week, and regular participation in competitive soccer games. Participants signed an informed consent in accordance with procedures approved by the local research ethics committee.

\section{Task and equipment}

The task was performed on a force platform (BTS, P6000, Italy),during which participants were tested while performing a power kick of a soccer ball (Figure 1). The kick was made with the upper face of the right foot, with the purpose of striking the ball with maximum power towards a rectangular target $(100 \mathrm{~cm} \times 60 \mathrm{~cm})$, placed on the ground $2 \mathrm{~m}$ in front of the ball position. The ball was stabilized on a base arranged beside the force 
platform. The force platform was used as support for the supporting leg during the kick. An additional (leveling) base was arranged behind and shifted to the left of the force platform as a starting point for kicking. The two support bases and force platform were leveled at 10 $\mathrm{cm}$ above the ground. The ball was stabilized on the support base by two elastic strings attached to fixed points on the floor. This device allowed the ball to move a few centimeters forward, without reaching the target, with a fast return to its original position. To begin the task, participants performed a single step to approach the ball, starting from the leveling base, and then kicking in a continuous action.

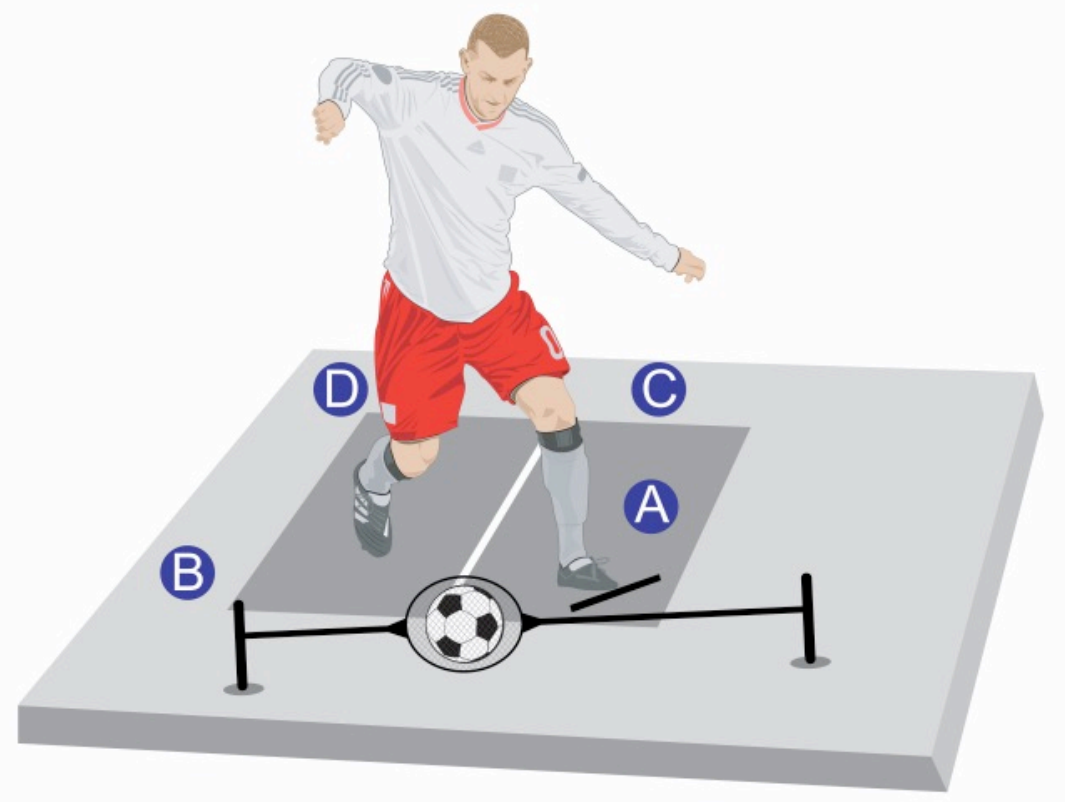

E

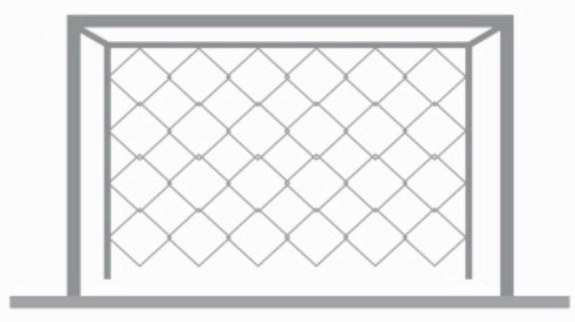

Figure 1. Representation of the soccer-specific task, showing (A) the locating foot placement of the support leg after the rapid approach step; (B) the attached ball that could be kicked with small displacement; (C) force plate for analysis of leg support data; (D) starting base; and (E) kick target.

Reflective markers (14 $\mathrm{mm}$ in diameter) were attached to the upper portion of the ball, and to the lateral malleolus of the kicking leg. These markers were tracked through four optoelectronic cameras (Vicon, model T10) to evaluate the time of contact of the kicking foot with the ball, the displacement of the kicking leg, and the ankle joint 
movements of the supporting leg. Wireless electromyographic electrodes (EMG, Delsys, Trigno) were used to record activation of the medial gastrocnemius (MG), tibial anterior $(T A)$, peroneus longus (PL), and soleus (SOL) muscles of the supporting leg. Force platform, kinematic, and EMG signals were synchronized through the Vicon Nexus system.

\section{Experimental design and procedures}

In a single group design, participants were evaluated immediately before and immediately after oscillatory movements of the swinging leg to induce muscle fatigue of the support leg. Three trials of the kicking task were performed in sequence, with inter-trial intervals of 10-15 s. Fatigue was induced by a protocol of voluntary swaying of the right (kicking) leg in the anteroposterior direction while maintaining unipedal support on the left leg. Oscillatory leg movements were generated predominantly by hip action, with arms free for body balance. Range of motion was individually defined, with peak-to-peak anteroposterior variation of approximately 1-1.2 m. The frequency of oscillatory movements was 80 cycles/min., following auditory signals emitted by a digital metronome (BOSS, model DB-60). Oscillatory movements were performed during intervals of $30 \mathrm{~s}$, interspersed with rest intervals (standing in bipedal support) of $10 \mathrm{~s}$. To prevent interruption of leg movements due to loss of balance, participants were allowed to lightly touch a fixed support in front of them in situations of high postural instability. Throughout the protocol, the participant's perception of effort was registered (scale ranging from 1-10). The fatigue induction phase was terminated when the participant reported being unable to continue the leg oscillatory movements because of fatigue-related discomfort. For functional assessment of fatigue, immediately before and immediately after the fatiguing activities, three maximal single leg vertical jumps were performed with the left leg.

EMG signals were sampled at a frequency of $2000 \mathrm{~Hz}$, amplified at a gain of 1000 and filtered with a bandpass of 20 to $400 \mathrm{~Hz}$. Center of pressure (CoP) position and kinematic data were sampled at a frequency of $200 \mathrm{~Hz}$ and filtered with a $10 \mathrm{~Hz}$ low pass Butterworth filter. Data were processed using Matlab software (MathWorks).

\section{Data analysis}

Analyzes were performed on the individual means of 3 trials. For the kicking task, the interval between the beginning of the vertical force increase on the force platform during the approach kicking step (identification criterion: vertical force equal to $1 \mathrm{~N}$ ) and the beginning of the displacement of the marker placed on the ball (foot-ball contact; identification criterion: marker speed equal to $20 \mathrm{~mm} / \mathrm{s}$ ) was analyzed.

The following dependent variables were analyzed: (a) mediolateral CoP displacement amplitude given by the peak-to-peak difference over the analyzed range (the mediolateral rather than the anteroposterior direction is considered to represent the main challenge to maintain a stable unipedal stance for kicking); (b) magnitude of muscle activation, defined by the mean square value of the electromyographic signal envelope, normalized by the mean value of the respective muscle in the pre-fatigue phase in quiet posture, calculated for the following intervals: $200 \mathrm{~ms}$ prior to foot-ball contact, and between 30-60 ms (short interval) and 80-135 ms (long interval) after foot-ball contact. These intervals were analyzed for all evaluated muscles; (c) co-contraction between the MG-TA and PL-TA muscles, given by the co-contraction index calculated by the temporal overlap of activation between the muscles during the analyzed intervals, from the 
electromyographic signal integral18; and (d) peak velocity of the kicking leg, given by the resulting maximum instantaneous velocity in the pre-contact interval.

Dependent variables were analyzed for comparisons between the pre- and postfatigue phases using the nonparametric Wilcoxon test to compare two repeated measures. The significance level was set at $5 \%$.

\section{RESULTS}

\section{Fatigue-induction protocol}

The duration of the protocol varied according to each participant, with a minimum of $67 \mathrm{~min}$. (100 trials of $30 \mathrm{~s}$ of leg swing) and maximum $140 \mathrm{~min}$. (210 trials). The average duration of the protocol among participants was $105 \mathrm{~min}$. (158 trials).

\section{Unipedal vertical jump}

Analysis of the unipedal vertical jump indicated that the air phase duration was significantly shorter in the post-fatigue phase $(M=310 \mathrm{~ms}$; $S E=20)$ compared to the prefatigue phase $(M=360 \mathrm{~ms} ; S E=10), z=1.99, p=0.04$.

\section{Kicking task}

Figure 2 presents signals from a representative trial in the pre-fatigue kicking task for the following: (A) kicking foot velocity, (B) mediolateral CoP, and (C) TA, (D) PL, (E) $M G$, and (F) SOL muscular activation. The analyzed time intervals (pre-contact, short and long post-contact) are represented by the shaded areas.

The results showed no significant differences between pre- and post-fatigue phases for kicking leg velocity (pre-fatigue, $M=8.13 \mathrm{~m} / \mathrm{s}$; $S E=0.55$; post-fatigue, $M=8.09$ $\mathrm{m} / \mathrm{s} ; S E=0.57$ ) or mediolateral CoP displacement amplitude of the support leg (pre-fatigue, $M=3.92 \mathrm{~cm} ; S E=0.96$; post fatigue, $M=3.97 \mathrm{~cm} ; S E=0.58$ ).

Pre-contact and short post-contact muscle activation analyzes indicated no fatigue effect in any evaluated muscles ( $z$ values $<1.78, p$ values $>0.05)$. Analysis of the long post-contact period indicated lower activation in the MG muscle $(z=2.20, p=0.02)$ and higher activation in the SOL muscle $(z=1.99, p=0.04)$ in the post-fatigue phase compared to the pre-fatigue phase (Figure 3 ). In the other muscles evaluated, no significant differences in muscle activation related to fatigue were found. However, at the descriptive level it is possible to observe a non-significant trend towards lower post-fatigue activation of the PL muscle in the pre-contact and short post-contact intervals. Similarly, it is possible to observe a tendency towards greater post-fatigue activation in the pre-contact interval of the TA and SOL muscles. Muscular fatigue failed to lead to significant effects on co-contraction ( $p$ values $>0.05$ ). 
Brazilian Journal of Motor Behavior

A

B

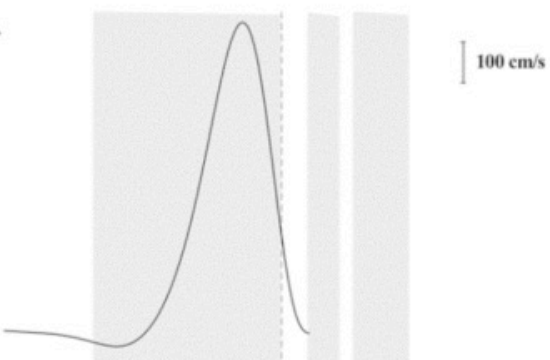

C

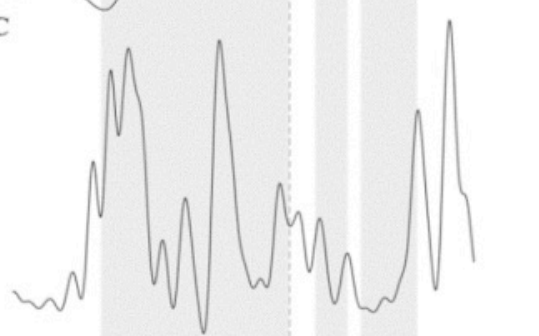

D

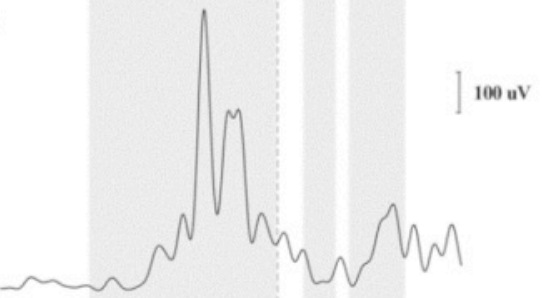

E

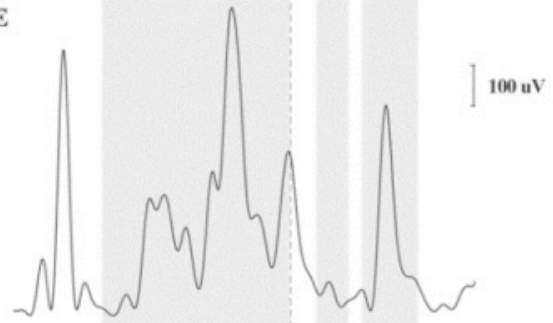

F

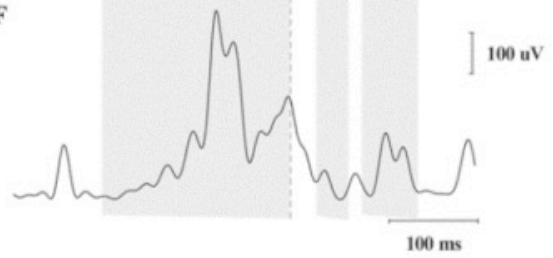

Figure 2. Representative curves of $(A)$ kicking foot velocity, $(B)$ mediolateral CoP displacement, and activation magnitude of the $(C)$ tibialis anterior $(T A),(D)$ peroneus longus $(P L),(E)$ medial gastrocnemius $(\mathrm{MG})$, and (F) soleus muscles (SOL). The vertical dashed line indicates the moment of foot-ball contact in the kick. The intervals in the analysis are shaded: pre-contact, short and long post-contact intervals. 
A
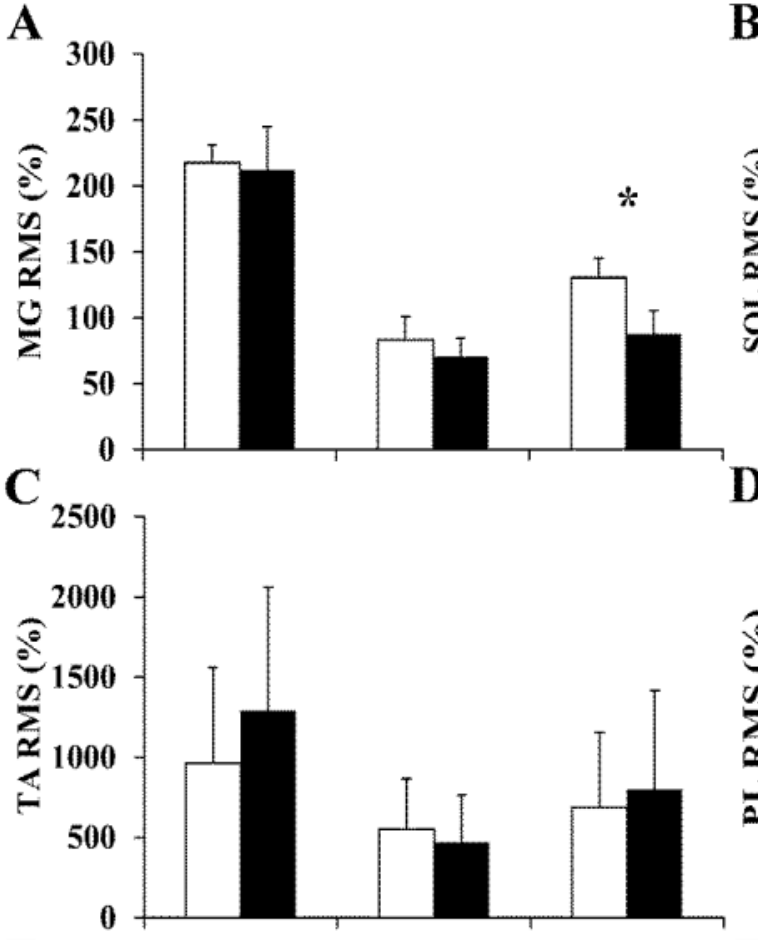

$\mathbf{E}$

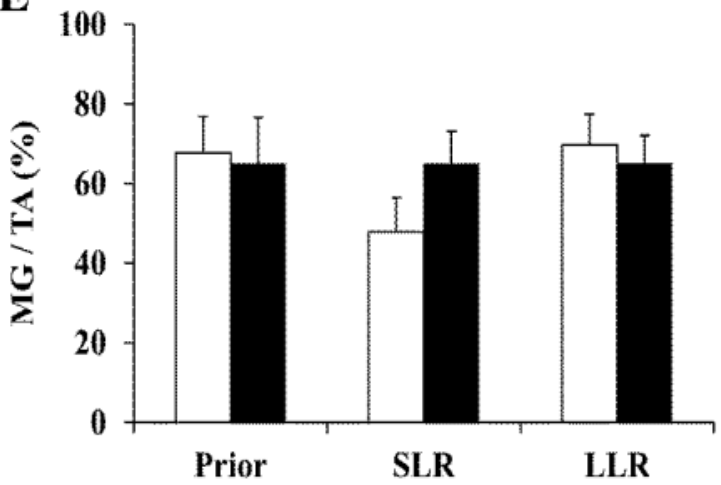

B $_{1000} \quad \square$ Pre-fatigue $\quad$ Post-fatigue

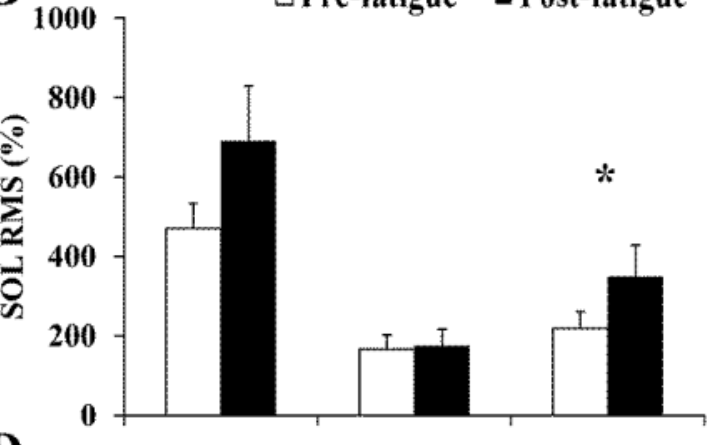

800

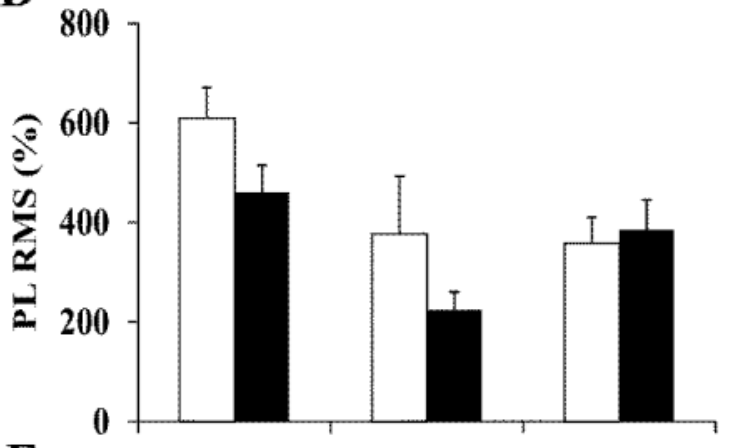

F

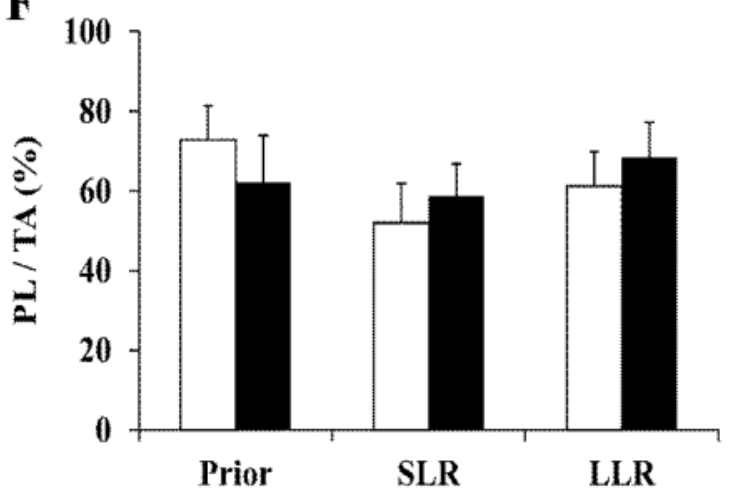

Figure 3. EMG during the kick: means of activation (standard error in vertical bars) of (A) medial gastrocnemius (MG), (B) soleus (SOL), (C) tibialis anterior (TA), and (D) peroneus longus (PL). * indicates statistically significant effects between pre- and post-fatigue.

\section{DISCUSSION}

The aim of the present study was to analyze the feasibility of evaluation of the effect of muscle fatigue of the support leg in soccer players on postural stability and activation of the stabilizing muscles of the ankle in the task of kicking a ball, using a similar motor pattern as in a real game situation, The protocol was shown to be effective in inducing fatigue, as indicated by the decreased performance in the functional assessment of a vertical jump in unipedal support, with mainly ankle plantar flexion (minimized hip action). Fatigue failed to lead to functional changes in either the support leg (based on CoP displacement) or kicking leg (based on peak velocity). The main changes in muscle 
activation occurred in the long post-contact period, with decreased MG muscle activation and increased SOL muscle activation.

Regarding the fatigue protocol employed, the exercise time was considerably different between participants. This fact may be related to the high interindividual variability of the responses found. In aerobic activity, Medbo et al. ${ }^{19}$ reported that the longer the duration of the treadmill exhaustion protocol, the greater the response variability. The protocol used in the present study was designed to cause local fatigue, with predominant involvement of lower and upper leg muscles, with repetitive swing leg movements requiring muscular contractions of the support leg to stabilize body posture. The long duration of the protocol indicates that the exercise was of relatively low intensity for the participants. This characteristic indicates that the protocol employed resembles the demands of the soccer game, with central fatigue characteristics, such as those caused by long-term running protocols on a treadmill ${ }^{12}$. Thus, it is possible to analyze the effect of a protocol that resembles the demands of the game on a specific task of the sport modality.

Analysis of the kicking task through peak kicking leg velocity suggests that the supporting leg muscle fatigue did not affect the kicking leg displacement. This result indicates that variations in muscle activation between the pre- and post-fatigue phases were not due to displacement with different kicking leg velocities between the pre- and post-fatigue phases. Similarly, no fatigue effect was found on the mediolateral stability of the supporting leg during kicking. Changes in postural and neuromuscular control strategies can be employed in an attempt to achieve the same level of stability after different fatigue protocols ${ }^{11-13}$ and ensure task efficiency. Baptista et al. ${ }^{20}$ showed that fatigue did not interfere with the accuracy of the kick in soccer players. However, to maintain accuracy (task efficiency) under fatigue, players increased their visual dependence, analyzed by the number and duration of visual fixations on areas of interest such as the ball and the target. Compensation strategies in the non-fatigued muscles, as suggested by the tendencies observed in the SOL and TA muscles, may have favored the maintenance of stability in the supporting leg during kicking.

When the individual is exposed to predictable perturbation (voluntary kick leg swing), one of the strategies used to maintain body stability is the production of anticipatory postural adjustments (APAs), such as pre-contact activation of postural muscles $^{21}$, by issuing descending commands from superior neural structures in anticipation of a postural perturbation $22-24$. After foot-ball contact, two moments of postcontact muscle response were considered, analogous to reactive responses (self-induced perturbation): short interval and long interval. According to Grüneberg et al. ${ }^{9}$, event-related fast muscular responses are predominantly modulated by the stretch reflex and long range responses. These appear to be task specific and modulated by a complex postural control system with corrective responses through the use of peripheral sensory information. Postural regulation during the kicking task is predominantly based on APAs. However, contact with the ball at the moment of kicking represents a sudden variation in the swinging leg displacement and can be considered an extrinsic perturbation, generating a possible reactive response in the supporting leg in an attempt to maintain postural balance. Analysis of the muscle activation results shows that the fatigue protocol mainly influenced the interval of supraspinal participation in postural control, in the long post-contact phase. Central fatigue causes the inability to voluntarily activate muscles as it affects excitability and synaptic input to motor neurons ${ }^{25}$, impairing the feedback processing of muscle 
spindles ${ }^{14}$. As a result, APAs and task-specific reactive responses could be affected in fatigued muscles.

During the execution of the kick the compensatory strategies that seem to have occurred between the stabilizing muscles of the ankle may have favored the maintenance of postural stability under fatigue. Since kicking is a soccer-specific task and players perform it under fatigue, these compensations may be adaptations developed by players as a result of soccer training. Previous results have shown that sport training induces specific adaptations in postural control to the modality practiced 26-28. Unpredictable situations that require reactive responses, such as ankle sprain situations, do not always allow the use of anticipatory mechanisms and, therefore, joint stabilization occurs after the perturbation, mainly through adjustments in the long latency phase of the muscular response $^{9}$. The post-contact compensatory adjustments observed in the present study appear to be task-dependent. Namely, they occur in specific tasks of the modality practiced as a result of specific training. Thus, it is possible to assume that in unpredictable situations of the modality these adjustments do not occur optimally, which may lead to increased instability at the ankle, increasing the risk of injury under muscular fatigue.

\section{CONCLUSION}

As the main conclusion, our study indicated the feasibility of evaluating the effect of muscular fatigue on dynamic balance and muscular activation in a power kick task. As far as we know, this is the first investigation to measure these variables in a context of mechanical requirements comparable to those seen in game-like situations in a laboratory setting. With respect to results interpretation, it is important to note that velocity of the kicking leg was not reduced after fatiguing activities of the support leg, allowing for appropriate comparisons between pre- and post-fatigue evaluations.

\section{REFERENCES}

1. Adlerton AK, Moritz U, Moe-Nilssen R. Forceplate and accelerometer measures for evaluating the effect of muscle fatigue on postural control during one-legged stance. Physiother Res Int. 2003;8(4):187-199.

2. Murphy DF, Connolly DA, Beynnon BD. Risk factors for lower extremity injury: a review of the literature. Br J Sports Med. 2003;37(1):13-29.

3. Ergen E, Ulkar B. Proprioception and ankle injuries in soccer. Clin Sports Med. 2008;27(1):195-217, x.

4. Hawkins RD, Hulse MA, Wilkinson C, Hodson A, Gibson M. The association football medical research programme: an audit of injuries in professional football. $\mathrm{Br} \mathrm{J}$ Sports Med. 2001;35(1):43-47.

5. Woods C, Hawkins R, Hulse M, Hodson A. The Football Association Medical Research Programme: an audit of injuries in professional football: an analysis of ankle sprains. $\mathrm{Br} \mathrm{J}$ Sports Med. 2003;37(3):233-238. 
6. McHugh MP, Tyler TF, Tetro DT, Mullaney MJ, Nicholas SJ. Risk factors for noncontact ankle sprains in high school athletes: the role of hip strength and balance ability. Am J Sports Med. 2006;34(3):464-470.

7. Gioftsidou AF, Malliou P, Pafis G, Beneka A, Godolias G. Effects of a soccer training session fatigue on balance ability. J Hum Sport Exerc. 2011;6(3):521-527.

8. Avela J, Kyrolainen $\mathrm{H}, \mathrm{Komi} \mathrm{PV}$. Altered reflex sensitivity after repeated and prolonged passive muscle stretching. J ApplPhysiol (1985). 1999;86(4):1283-1291.

9. Gruneberg C, Nieuwenhuijzen PH, Duysens J. Reflex responses in the lower leg following landing impact on an inverting and non-inverting platform. J Physiol. 2003;550(Pt 3):985993.

10. Taylor JL, Todd G, Gandevia SC. Evidence for asupraspinal contribution to human muscle fatigue. ClinExpPharmacol Physiol. 2006;33(4):400-405.

11. Paillard T. Effects of general and local fatigue on postural control: a review. NeurosciBiobehav Rev. 2012;36(1):162-176.

12. Boyas $S$, Remaud $A$, Rivers $E$, Bilodeau M. Fatiguing exercise intensity influences the relationship between parameters reflecting neuromuscular function and postural control variables. PLoS One. 2013;8(8):e72482.

13. Ritzmann R, Freyler K, Werkhausen A, Gollhofer A. Changes in Balance Strategy and Neuromuscular Control during a Fatiguing Balance Task-A Study in Perturbed Unilateral Stance. Front Hum Neurosci. 2016;10:289.

14. Taylor JL, Amann M, Duchateau J, Meeusen R, Rice CL. Neural Contributions to Muscle Fatigue: From the Brain to the Muscle and Back Again. Med Sci Sports Exerc. 2016;48(11):2294-2306.

15. Greig M, Walker-Johnson $C$. The influence of soccer-specific fatigue on functional stability. PhysTher Sport. 2007;7(4):185-190.

16. Arliani GG, Almeida GP, Dos Santos CV, Venturini AM, AsturDda C, Cohen M. The effects of exertion on the postural stability in young soccer players. ActaOrtop Bras. 2013;21(3):155-158.

17. Pau M, Ibba G, Attene G. Fatigue-induced balance impairment in young soccer players. J Athl Train. 2014;49(4):454-461.

18. Nagai K, Yamada M, Uemura K, Yamada Y, Ichihashi N, Tsuboyama T. Differences in muscle coactivation during postural control between healthy older and young adults. Arch GerontolGeriatr. 2011;53(3):338-343.

19. Medbo JI, Mohn AC, Tabata I, Bahr R, Vaage O, Sejersted OM. Anaerobic capacity determined by maximal accumulated 02 deficit. J ApplPhysiol (1985). 1988;64(1):50-60.

20. Baptista AM, Santiago PRP, Faria MH, Simieli L, Barbieri FA. Efeito da fadiga na percepção e na precisão durante o chute com a bola parada no futsal utilizando a estratégia de goleiro-dependente. IX CongressoBrasileiro de Comportamento Motor; 2018.

21. Aruin AS. The organization of anticipatory postural adjustments. J Aut Cont. 2002;12(1):31-37. 
22. Horak FB, Nashner LM. Central programming of postural movements: adaptation to altered support-surface configurations. J Neurophysiol. 1986;55(6):1369-1381.

23. Horak FB, Diener HC, Nashner LM. Influence of central set on human postural responses. J Neurophysiol. 1989;62(4):841-853.

24. Horak FB, Nutt JG, Nashner LM. Postural inflexibility in parkinsonian subjects. J Neurol Sci. 1992;111(1):46-58.

25. Goodall S, Howatson G, Thomas K. Modulation of specific inhibitory networks in fatigued locomotor muscles of healthy males. Exp Brain Res. 2018;236(2):463-473.

26. Paillard T, Noe F, Riviere T, Marion V, Montoya R, Dupui P. Postural performance and strategy in the unipedal stance of soccer players at different levels of competition. J Athl Train. 2006;41(2):172-176.

27. Freyler K, Krause A, Gollhofer A, Ritzmann R. Specific Stimuli Induce Specific Adaptations: Sensorimotor Training vs. Reactive Balance Training. PLoS One. 2016;11(12):e0167557.

28. Paillard T. Plasticity of the postural function to sport and/or motor experience. NeurosciBiobehav Rev. 2017;72:129-152.

Citation: Ávila de Oliveira J, Ribeiro de Souza C, Rinaldin CDP, Coelho DB, Teixeira LA. Feasibility of evaluating effects of muscle fatigue on postural stability and muscular activation of the supporting leg in the soccer power kicking. BJMB. 2019:13(5): 144-154.

Editors: Dr Fabio Augusto Barbieri - São Paulo State University (UNESP), Bauru, SP, Brazil; Dr José Angelo Barela São Paulo State University (UNESP), Rio Claro, SP, Brazil; Dr Natalia Madalena Rinaldi - Federal University of Espírito Santo (UFES), Vitória, ES, Brazil.

Copyright:@ 2019 Ávila de Oliveira, Ribeiro de Souza, Rinaldin, Coelho and Teixeira and BJMB. This is an openaccess article distributed under the terms of the Creative Commons Attribution- Non Commercial- No Derivatives 4.0 International License which permits unrestricted use, distribution, and reproduction in any medium, provided the original author and source are credited.

Funding: This work was supported by the Brazilian Council of Science and Technology (CNPq, grant number 435412/2018-3, to LAT; grant number133659/2019-4, to JAO). 